\title{
Non-perturbative construction of the Luttinger-Ward functional
}

\author{
M.Potthoff \\ Institut für Theoretische Physik und Astrophysik, Universität Würzburg, \\ Am Hubland, D-97074 Würzburg, Germany
}

Received February 10, 2006

For a system of correlated electrons, the Luttinger-Ward functional provides a link between static thermodynamic quantities on the one hand and single-particle excitations on the other. The functional is useful in deriving several general properties of the system as well as in formulating the thermodynamically consistent approximations. Its original construction, however, is perturbative as it is based on the weak-coupling skeleton-diagram expansion. Here, it is shown that the Luttinger-Ward functional can be derived within a general functional-integral approach. This alternative and non-perturbative approach stresses the fact that the Luttinger-Ward functional is universal for a large class of models.

Key words: Luttinger-Ward functional, conserving approximations, diagrammatic perturbation theory, functional-integral approach, dynamical mean-field theory, self-energy-functional theory

PACS: $71.10 .-w, 71.15 .-m$

\section{Introduction}

For a system of correlated electrons in equilibrium, there are several relations [1-3] between static quantities which describe the thermodynamics of the system and dynamic quantities which describe its one-particle excitations. Static quantities are given by the grand potential $\Omega$ and its derivatives with respect to temperature $T$, chemical potential $\mu$ etc. The one-electron Green's function $\mathbf{G}=\mathbf{G}\left(\mathrm{i} \omega_{n}\right)$ or the self-energy $\boldsymbol{\Sigma}=\boldsymbol{\Sigma}\left(\mathrm{i} \omega_{n}\right)$, on the other hand, are dynamic quantities which yield (equivalent) information on an idealized (photoemission or inverse photoemission) excitation process.

The Luttinger-Ward functional $\widehat{\Phi}[\mathbf{G}]$ provides a special relation between static and dynamic quantities with several important properties [4]: Firstly, the grand potential is obtained from the Luttinger-Ward functional evaluated at the exact Green's function, $\Phi=\widehat{\Phi}[\mathbf{G}]$, via

$$
\Omega=\Phi+\operatorname{Tr} \ln \mathbf{G}-\operatorname{Tr} \mathbf{\Sigma} \mathbf{G} .
$$

Secondly, the functional derivative of the Luttinger-Ward functional,

$$
\frac{1}{T} \frac{\delta \widehat{\Phi}[\mathbf{G}]}{\delta \mathbf{G}}=\widehat{\mathbf{\Sigma}}[\mathbf{G}],
$$

defines a functional $\widehat{\boldsymbol{\Sigma}}[\mathbf{G}]$ which gives the exact self-energy of the system if evaluated at the exact Green's function. The relation $\boldsymbol{\Sigma}=\widehat{\boldsymbol{\Sigma}}[\mathbf{G}]$ is independent of the Dyson equation $\mathbf{G}^{-1}=\mathbf{G}_{0}^{-1}-\mathbf{\Sigma}$. Thirdly, in the non-interacting limit:

$$
\widehat{\Phi}[\mathbf{G}] \equiv 0 \quad \text { for } \quad \mathbf{U}=0 .
$$

Finally, the functional dependence $\widehat{\Phi}[\mathbf{G}]$ is completely determined by the interaction part of the Hamiltonian and independent of the one-particle part:

$$
\widehat{\Phi}[\mathbf{G}] \text { universal . }
$$


This universality property can also be expressed as follows: Two systems with the same interaction $\mathbf{U}$ but different one-particle parameters $\mathbf{t}$ (on-site energies and hopping integrals) in the respective Hamiltonian are described by the same Luttinger-Ward functional. Using equation (2), this implies that the functional $\widehat{\boldsymbol{\Sigma}}[\mathbf{G}]$ is universal, too.

In [4] it is shown by Luttinger and Ward that $\widehat{\Phi}[\mathbf{G}]$ can be constructed order by order in diagrammatic weak-coupling perturbation theory. $\Phi$ is obtained as the limit of the infinite series of closed diagrams without any self-energy insertions and with all free propagators in a diagram replaced by fully interacting ones (see figure 1). Generally, this skeleton-diagram expansion cannot be summed up to get a closed form for $\widehat{\Phi}[\mathbf{G}]$. So, unfortunately, the explicit functional dependence $\widehat{\Phi}[\mathbf{G}]$ is actually unknown - even for the most simple Hamiltonians such as the Hubbard model [5]. The defining properties, equations (1)-(4), however, are easily verified [4].

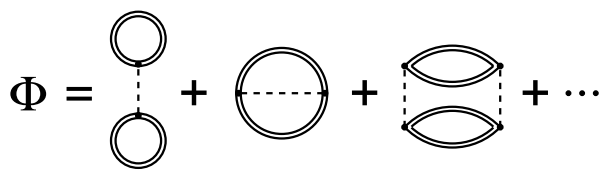

Figure 1. Classical definition of the Luttinger-Ward functional $\widehat{\Phi}[\mathbf{G}]$. Double lines: fully interacting propagator G. Dashed lines: interaction $\mathbf{U}$.

The Luttinger-Ward functional is useful for several general considerations: With the help of $\widehat{\Phi}[\mathbf{G}]$ and the Dyson equation, the grand potential can be considered as a functional of the Green's function $\Omega=\widehat{\Omega}[\mathbf{G}]$ or as functional of the self-energy $\Omega=\widehat{\Omega}[\boldsymbol{\Sigma}]$, such that $\Omega$ is stationary at the physical $\mathbf{G}$ or $\boldsymbol{\Sigma}[4,6]$. This represents a remarkable variational principle which connects static physical quantities with dynamic ones. The Luttinger-Ward functional is also used in the microscopic derivation of some zero- or low-temperature properties of Fermi liquids as discussed in [4,7]. The derivative of the functional, equation (2), shows the self-energy to be gradient field when considered as a functional of the Green's function, $\widehat{\mathbf{\Sigma}}[\mathbf{G}]$. This fact is related to certain symmetry properties of two-particle Green's functions as originally noted by Baym and Kadanoff [8]. Furthermore, the Luttinger-Ward functional is of great importance in the construction of thermodynamically consistent approximations. The so-called conserving (" $\Phi$-derivable") approximations virtually start from the Luttinger-Ward functional $[6,8]$. This is essential in order to prove that these approximations obey a number of macroscopic conservation laws. The Hartree-Fock and the random-phase approximations are well-known examples. These "classical" conserving approximations as well as higher-order approaches such as the fluctuation-exchange approximation (FLEX) $[9,10]$ are essentially limited to the weak-coupling regime. In the strong-coupling regime, the noncrossing approximation (NCA) [11,12] and different generalizations (see e. g. [13]) are $\Phi$-derivable. However, the Luttinger-Ward functional can also be used in constructing non-perturbative approximations. This was first realized in the context of the dynamical mean-field theory (DMFT) for lattice models of correlated electrons [14-17]. Here, one exploits the universality of the functional, equation (4), to achieve an (approximate) mapping of the original lattice model onto a simpler impurity model with the same interaction part. The fact that $\widehat{\Phi}[\mathbf{G}]$ is the same for a large class of systems, has recently been shown $[18,19]$ to be the key feature that makes it possible to construct several non-perturbative and thermodynamically consistent approximations [20-22]. This idea has been termed "self-energy-functional approach" (SFA).

Such general considerations remain valid as long as the Luttinger-Ward functional is well defined. This presupposes that the skeleton-diagram expansion is convergent or at least that formal manipulations of diagrammatic quantities are consistent in themselves and eventually lead to physically meaningful results. Provided that one can assure that no singular point is passed when starting from the non-interacting Fermi gas and increasing the interaction strength, this seems to be plausible. A strict proof that the skeleton-diagram expansion is well-behaved, however, will hardly be possible in most concrete situations. On the contrary, it is well known that the expansion is questionable in a number of cases, e. g. in case of a symmetry-broken state or a state that is 
not "adiabatically connected" to the non-interacting limit, such as a Mott insulator. The skeletondiagram expansion may break down even in the absence of any spontaneous symmetry breaking in a (strongly correlated) state that gradually evolves from a metallic Fermi liquid. This has explicitly been shown by Hofstetter and Kehrein [23] for the narrow-band limit of the single-impurity Anderson model (see [24,25] for a discussion of possible physical consequences). Generally speaking there is no strict argument available that ensures the convergence of the skeleton-diagram expansion in the strong-coupling regime.

The purpose of the present paper is to show that a construction of the Luttinger-Ward functional is possible without making use of the skeleton-diagram expansion. The proposed construction is based on a standard functional-integral approach and avoids the formal complications mentioned above. Thereby, one achieves an alternative and in particular non-perturbative route to the general properties of correlated electron systems derived from the functional, to the dynamical mean-field theory as well as to the self-energy-functional approach. It should be stressed that the intended construction of the Luttinger-Ward functional requires more than a simple definition of the quantity $\Phi$ (which could trivially be achieved by using equation (1): $\Phi \equiv \Omega-\operatorname{Tr} \ln \mathbf{G}+\operatorname{Tr} \boldsymbol{\Sigma} \mathbf{G}$ ). The task is rather to provide a functional $\widehat{\Phi}[\mathbf{G}]$ with the properties $(1)-(4)$.

Previous approaches are either perturbative or are incapable of proving equations (1)-(4): A construction of the Luttinger-Ward functional different from the original one [4] has been given by Baym [6]: The existence of $\widehat{\Phi}[\mathbf{G}]$ is deduced from a "vanishing curl condition", $\delta \Sigma\left(1,1^{\prime}\right) / \delta G\left(2^{\prime}, 2\right)=$ $\delta \Sigma\left(2,2^{\prime}\right) / \delta G\left(1^{\prime}, 1\right)$, which is derived from an analysis of the functional dependence of $\mathbf{G}$ on an arbitrary (time-dependent) external perturbation $\mathbf{J}$. Moreover, an independent functional relation $\boldsymbol{\Sigma}=\widehat{\boldsymbol{\Sigma}}[\mathbf{G}]$ is required. In [6] the latter is assumed to be given by the (full or by a truncated) skeleton-diagram expansion, and consequently this approach is perturbative again.

As shown in [6], the Green's function in the presence of an external field $\mathbf{J}$ can be derived from the grand potential $\widehat{\Omega}^{\prime}[\mathbf{J}]$ as $\widehat{\mathbf{G}}(\mathbf{J})=(1 / T) \delta \widehat{\Omega}^{\prime}[\mathbf{J}] / \delta \mathbf{J}$. Using the inverse functional, $\widehat{\mathbf{J}}[\mathbf{G}]$, Legendre transformation yields $\widehat{\Omega}[\mathbf{G}]=\widehat{\Omega}^{\prime}[\widehat{\mathbf{J}}[\mathbf{G}]]-\operatorname{Tr} \mathbf{G} \widehat{\mathbf{J}}[\mathbf{G}]$. This (non-perturbative) functional and the Dyson equation can be used to define $\widehat{\Phi}[\mathbf{G}] \equiv \widehat{\Omega}[\mathbf{G}]-\operatorname{Tr} \ln \mathbf{G}+\operatorname{Tr}\left(\mathbf{G}_{0}^{-1}-\mathbf{G}^{-1}\right) \mathbf{G}$. This idea is in the spirit of the effective action approach $[26,27]$. Here, however, the problem is that the universality of $\widehat{\Phi}[\mathbf{G}]$, equation (4), cannot be proven. The Luttinger-Ward functional constructed in this way explicitly depends on $\mathbf{G}_{0}$ and hence on the one-particle parameters $\mathbf{t}$.

The paper is organized as follows: The next section briefly introduces the notations and the quantities of interest. The construction of the Luttinger-Ward functional is described in section 3 . Section 4 gives a brief discussion of the properties of the functional and its use within the dynamical mean-field theory and the self-energy-functional approach. The results are summed up in section 5 .

\section{Static and dynamic quantities}

Consider a system of electrons at temperature $T$ and chemical potential $\mu$ in thermal equilibrium and let $H=H(\mathbf{t}, \mathbf{U})=H_{0}(\mathbf{t})+H_{1}(\mathbf{U})$ be its Hamiltonian where

$$
H_{0}(\mathbf{t})=\sum_{\alpha \beta} t_{\alpha \beta} c_{\alpha}^{\dagger} c_{\beta}, \quad H_{1}(\mathbf{U})=\frac{1}{2} \sum_{\alpha \beta \gamma \delta} U_{\alpha \beta \delta \gamma} c_{\alpha}^{\dagger} c_{\beta}^{\dagger} c_{\gamma} c_{\delta} .
$$

An index $\alpha$ refers to an arbitrary set of quantum numbers characterizing a one-particle basis state. If $N$ is the total particle-number operator, the grand potential of the system is given by $\Omega_{\mathbf{t}, \mathbf{U}}=-T \ln Z_{\mathbf{t}, \mathbf{U}}$ where

$$
Z_{\mathbf{t}, \mathbf{U}}=\operatorname{tr} \exp (-(H(\mathbf{t}, \mathbf{U})-\mu N) / T)
$$

is the partition function. Here and in the following the dependence of all quantities on the oneparticle parameters $\mathbf{t}$ and the interaction parameters $\mathbf{U}$ is made explicit through the subscripts.

Using a matrix notation, the free one-particle Green's function is denoted by $\mathbf{G}_{\mathbf{t}, 0}$. Its elements (for fixed $\mu$ ) are given by:

$$
G_{\mathbf{t}, 0, \alpha \beta}\left(\mathrm{i} \omega_{n}\right)=\left(\frac{1}{\mathrm{i} \omega_{n}+\mu-\mathbf{t}}\right)_{\alpha \beta} .
$$


Here $i \omega_{n}=\mathrm{i}(2 n+1) \pi T$ is the $n$-th Matsubara frequency. The fully interacting Green's function is denoted by $\mathbf{G}_{\mathbf{t}, \mathbf{U}}$. Using Grassmann variables $\xi_{\alpha}\left(\mathrm{i} \omega_{n}\right)=T^{1 / 2} \int_{0}^{1 / T} \mathrm{~d} \tau \mathrm{e}^{\mathrm{i} \omega_{n} \tau} \xi_{\alpha}(\tau)$ and $\xi_{\alpha}^{*}\left(\mathrm{i} \omega_{n}\right)=$ $T^{1 / 2} \int_{0}^{1 / T} \mathrm{~d} \tau \mathrm{e}^{-\mathrm{i} \omega_{n} \tau} \xi_{\alpha}^{*}(\tau)$, its elements can be written as [3]

$$
G_{\mathbf{t}, \mathbf{U}, \alpha \beta}\left(\mathrm{i} \omega_{n}\right)=-\left\langle\xi_{\alpha}\left(\mathrm{i} \omega_{n}\right) \xi_{\beta}^{*}\left(\mathrm{i} \omega_{n}\right)\right\rangle_{\mathbf{t}, \mathbf{U}}=\frac{-1}{Z_{\mathbf{t}, \mathbf{U}}} \int D \xi D \xi^{*} \xi_{\alpha}\left(\mathrm{i} \omega_{n}\right) \xi_{\beta}^{*}\left(\mathrm{i} \omega_{n}\right) \exp \left(A_{\mathbf{t}, \mathbf{U}, \xi \xi^{*}}\right),
$$

where

$$
A_{\mathbf{t}, \mathbf{U}, \xi \xi^{*}}=\sum_{n, \alpha \beta} \xi_{\alpha}^{*}\left(\mathrm{i} \omega_{n}\right)\left(\left(\mathrm{i} \omega_{n}+\mu\right) \delta_{\alpha \beta}-t_{\alpha \beta}\right) \xi_{\beta}\left(\mathrm{i} \omega_{n}\right)-\frac{1}{2} \sum_{\alpha \beta \gamma \delta} U_{\alpha \beta \delta \gamma} \int_{0}^{1 / T} \mathrm{~d} \tau \xi_{\alpha}^{*}(\tau) \xi_{\beta}^{*}(\tau) \xi_{\gamma}(\tau) \xi_{\delta}(\tau)
$$

is the action. Finally, the self-energy is defined as

$$
\boldsymbol{\Sigma}_{\mathbf{t}, \mathbf{U}}=\mathbf{G}_{\mathbf{t}, 0}^{-1}-\mathbf{G}_{\mathbf{t}, \mathbf{U}}^{-1}
$$

The goal is to construct a functional $\widehat{\Phi}_{\mathbf{U}}[\mathbf{G}]$ (where $\mathbf{G}$ is considered to be a free variable) which vanishes in the non-interacting case, $\widehat{\Phi}_{0}[\mathbf{G}]=0$ [equation (3)], which is universal, i. e. independent of $\mathbf{t}$ [equation (4)], which yields $\widehat{\Phi}_{\mathbf{U}}\left[\mathbf{G}_{\mathbf{t}, \mathbf{U}}\right]=\Omega_{\mathbf{t}, \mathbf{U}}-\operatorname{Tr} \ln \mathbf{G}_{\mathbf{t}, \mathbf{U}}+\operatorname{Tr}\left(\boldsymbol{\Sigma}_{\mathbf{t}, \mathbf{U}} \mathbf{G}_{\mathbf{t}, \mathbf{U}}\right)$ if evaluated at the exact Green's function $\mathbf{G}=\mathbf{G}_{\mathbf{t}, \mathbf{U}}$ [equation (1)], and the derivative of which is a functional $\widehat{\boldsymbol{\Sigma}}[\mathbf{G}]$ with $\widehat{\boldsymbol{\Sigma}}\left[\mathbf{G}_{\mathbf{t}, \mathbf{U}}\right]=\boldsymbol{\Sigma}_{\mathbf{t}, \mathbf{U}}$ [equation (2)]. (Here the notation $\operatorname{Tr} \mathbf{A} \equiv T \sum_{n} \sum_{\alpha} \mathrm{e}^{\mathrm{i} \omega_{n} 0^{+}} A_{\alpha \alpha}\left(\mathrm{i} \omega_{n}\right)$ is used. $0^{+}$is a positive infinitesimal. Functionals $\widehat{A}=\widehat{A}[\cdots]$ are indicated by a hat and should be distinguished clearly from physical quantities $A$.)

For the classical construction of $\widehat{\Phi}_{\mathbf{U}}[\mathbf{G}]$ via the skeleton-diagram expansion (figure 1), these properties are easily verified: The universality of the functional [equation (4)] is obvious as any diagram depends on $\mathbf{U}$ and on $\mathbf{G}$ only; there is no explicit dependence on the free Green's function $\mathbf{G}_{\mathbf{t}, 0}$, i. e. no explicit dependence on $\mathbf{t}$. Since there is no zeroth-order diagram, $\widehat{\Phi}_{\mathbf{U}}[\mathbf{G}]$ trivially vanishes for $\mathbf{U}=0$ [equation (3)]. The functional derivative of $\widehat{\Phi}[\mathbf{G}$ ] with respect to $\mathbf{G}$ corresponds to the removal of a propagator from each of the $\Phi$ diagrams. Taking care of topological factors $[1,4]$, one ends up with the skeleton-diagram expansion for the self-energy, i. e. one gets equation (2). Using equation (2), the Dyson equation (10), and $\Phi_{\mathbf{t}, \mathbf{U}} \equiv \widehat{\Phi}_{\mathbf{U}}\left[\mathbf{G}_{\mathbf{t}, \mathbf{U}}\right]$, the $\mu$ derivative of the l.h.s. and of the r.h.s of equation (1) are equal for any fixed interaction strength $\mathbf{U}$ and temperature $T$. Namely, $(\partial / \partial \mu)\left(\Phi_{\mathbf{t}, \mathbf{U}}+\operatorname{Tr} \ln \mathbf{G}_{\mathbf{t}, \mathbf{U}}-\operatorname{Tr} \boldsymbol{\Sigma}_{\mathbf{t}, \mathbf{U}} \mathbf{G}_{\mathbf{t}, \mathbf{U}}\right)=\operatorname{Tr} \mathbf{G}_{\mathbf{t}, \mathbf{U}}^{-1}\left(\partial \mathbf{G}_{\mathbf{t}, \mathbf{U}} / \partial \mu\right)-\operatorname{Tr} \mathbf{G}_{\mathbf{t}, \mathbf{U}}\left(\partial \boldsymbol{\Sigma}_{\mathbf{t}, \mathbf{U}} / \partial \mu\right)=$ $-\operatorname{Tr} \mathbf{G}_{\mathbf{t}, \mathbf{U}}=-\langle N\rangle_{\mathbf{t}, \mathbf{U}}=\partial \Omega_{\mathbf{t}, \mathbf{U}} / \partial \mu$. Integration over $\mu$ yields equation (1). (Note that equation (1) trivially holds for $\mu \rightarrow-\infty$, i. e. for $\langle N\rangle_{\mathbf{t}, \mathbf{U}} \rightarrow 0$ since $\boldsymbol{\Sigma}_{\mathbf{t}, \mathbf{U}}=0$ and $\Phi_{\mathbf{t}, \mathbf{U}}=0$ in this limit). An equivalent derivation of equation (1) can be given by a coupling-constant integration [4].

\section{Luttinger-Ward functional}

The starting point is the standard functional-integral representation of the partition function as given in [3], for example: Define the functional

$$
\widehat{\Omega}_{\mathbf{U}}\left[\mathbf{G}_{0}^{-1}\right]=-T \ln \widehat{Z}_{\mathbf{U}}\left[\mathbf{G}_{0}^{-1}\right]
$$

with

$$
\widehat{Z}_{\mathbf{U}}\left[\mathbf{G}_{0}^{-1}\right]=\int D \xi D \xi^{*} \exp \left(\widehat{A}_{\mathbf{U}, \xi \xi^{*}}\left[\mathbf{G}_{0}^{-1}\right]\right)
$$

and

$$
\widehat{A}_{\mathbf{U}, \xi \xi^{*}}\left[\mathbf{G}_{0}^{-1}\right]=\sum_{n, \alpha \beta} \xi_{\alpha}^{*}\left(\mathrm{i} \omega_{n}\right) G_{0, \alpha \beta}^{-1}\left(\mathrm{i} \omega_{n}\right) \xi_{\beta}\left(\mathrm{i} \omega_{n}\right)-\frac{1}{2} \sum_{\alpha \beta \gamma \delta} U_{\alpha \beta \delta \gamma} \int_{0}^{1 / T} \mathrm{~d} \tau \xi_{\alpha}^{*}(\tau) \xi_{\beta}^{*}(\tau) \xi_{\gamma}(\tau) \xi_{\delta}(\tau)
$$


$\widehat{\Omega}_{\mathbf{U}}\left[\mathbf{G}_{0}^{-1}\right]$ parametrically depends on $\mathbf{U} \cdot \mathbf{G}_{0}^{-1}$ is considered to be a free variable. At the (matrix inverse of the) exact free Green's function, $\mathbf{G}_{0}^{-1}=\mathbf{G}_{\mathbf{t}, 0}^{-1}$, the functional yields the exact grand potential,

$$
\widehat{\Omega}_{\mathbf{U}}\left[\mathbf{G}_{\mathbf{t}, 0}^{-1}\right]=\Omega_{\mathbf{t}, \mathbf{U}}
$$

of the system with Hamiltonian $H=H_{0}(\mathbf{t})+H_{1}(\mathbf{U})$. Its derivative defines a functional $\widehat{\mathbf{g}}_{\mathbf{U}}\left[\mathbf{G}_{0}^{-1}\right]$,

$$
\frac{1}{T} \frac{\delta \widehat{\Omega}_{\mathbf{U}}\left[\mathbf{G}_{0}^{-1}\right]}{\delta \mathbf{G}_{0}^{-1}}=-\frac{1}{\widehat{Z}_{\mathbf{U}}\left[\mathbf{G}_{0}^{-1}\right]} \frac{\delta \widehat{Z}_{\mathbf{U}}\left[\mathbf{G}_{0}^{-1}\right]}{\delta \mathbf{G}_{0}^{-1}} \equiv-\widehat{\mathbf{g}}_{\mathbf{U}}\left[\mathbf{G}_{0}^{-1}\right],
$$

with the property

$$
\widehat{\mathrm{g}}_{\mathbf{U}}\left[\mathbf{G}_{\mathbf{t}, 0}^{-1}\right]=\mathbf{G}_{\mathbf{t}, \mathbf{U}}
$$

which is easily verified using equation (8).

The strategy to be pursued is the following: $\widehat{\mathrm{g}}_{\mathbf{U}}\left[\mathbf{G}_{0}^{-1}\right]$ is a universal (t independent) functional and can be used in order to construct a universal relation $\mathbf{G}=\widehat{\mathbf{G}}_{\mathbf{U}}[\boldsymbol{\Sigma}]$ between the one-particle Green's function and the self-energy independent of the Dyson equation. Using the universal functionals $\widehat{\Omega}_{\mathbf{U}}\left[\mathbf{G}_{0}^{-1}\right]$ and $\widehat{\mathbf{G}}_{\mathbf{U}}[\boldsymbol{\Sigma}]$, a universal functional $\widehat{F}_{\mathbf{U}}[\boldsymbol{\Sigma}]$ is defined the derivative of which essentially yields $\widehat{\mathbf{G}}_{\mathbf{U}}[\boldsymbol{\Sigma}]$. The Luttinger-Ward functional can be obtained by Legendre transformation and is universal in construction.

To start with, consider the equation

$$
\widehat{\mathrm{g}}_{\mathbf{U}}\left[\mathbf{G}^{-1}+\mathbf{\Sigma}\right]=\mathbf{G} \text {. }
$$

This is a relation between the variables $\mathbf{G}$ and $\boldsymbol{\Sigma}$ which, for a given $\boldsymbol{\Sigma}$, may be solved for $\mathbf{G}$. This defines a functional $\widehat{\mathbf{G}}_{\mathbf{U}}[\boldsymbol{\Sigma}]$, i. e.

$$
\widehat{\mathrm{g}}_{\mathrm{U}}\left[\widehat{\mathrm{G}}_{\mathbf{U}}[\boldsymbol{\Sigma}]^{-1}+\boldsymbol{\Sigma}\right]=\widehat{\mathbf{G}}_{\mathbf{U}}[\boldsymbol{\Sigma}]
$$

For a given self-energy $\boldsymbol{\Sigma}$, the Green's function $\mathbf{G}=\widehat{\mathbf{G}}_{\mathbf{U}}[\boldsymbol{\Sigma}]$ is defined to be the solution of equation (17). From the Dyson equation (10) and equation (16) it is obvious that the relation (17) is satisfied for $\mathbf{G}$ and $\boldsymbol{\Sigma}$ being the exact Green's function and the exact self-energy, $\mathbf{G}=\mathbf{G}_{\mathbf{t}, \mathbf{U}}$ and $\boldsymbol{\Sigma}=\boldsymbol{\Sigma}_{\mathbf{t}, \mathbf{U}}$, of a system with the interaction $\mathbf{U}$ and some set of one-particle parameters $\mathbf{t}$ $\left(H=H_{0}(\mathbf{t})+H_{1}(\mathbf{U})\right)$. Hence,

$$
\widehat{\mathbf{G}}_{\mathbf{U}}\left[\boldsymbol{\Sigma}_{\mathbf{t}, \mathbf{U}}\right]=\mathbf{G}_{\mathbf{t}, \mathbf{U}} \text {. }
$$

A brief discussion of the existence and the uniqueness of possible solutions of the relation (17) is given in Appendix A.

Using the functionals $\widehat{\Omega}_{\mathbf{U}}\left[\mathbf{G}_{0}^{-1}\right]$ and $\widehat{\mathbf{G}}_{\mathbf{U}}[\boldsymbol{\Sigma}]$, a functional $\widehat{F}_{\mathbf{U}}[\boldsymbol{\Sigma}]$ can be defined as:

$$
\widehat{F}_{\mathbf{U}}[\boldsymbol{\Sigma}]=\widehat{\Omega}_{\mathbf{U}}\left[\widehat{\mathbf{G}}_{\mathbf{U}}[\boldsymbol{\Sigma}]^{-1}+\boldsymbol{\Sigma}\right]-\operatorname{Tr} \ln \widehat{\mathbf{G}}_{\mathbf{U}}[\boldsymbol{\Sigma}] .
$$

Using equation (15) one finds:

$$
\frac{1}{T} \frac{\delta \widehat{F}_{\mathbf{U}}[\boldsymbol{\Sigma}]}{\delta \boldsymbol{\Sigma}}=-\widehat{\mathbf{g}}_{\mathbf{U}}\left[\widehat{\mathbf{G}}_{\mathbf{U}}[\boldsymbol{\Sigma}]^{-1}+\boldsymbol{\Sigma}\right] \cdot\left(\frac{\delta \widehat{\mathbf{G}}_{\mathbf{U}}[\boldsymbol{\Sigma}]^{-1}}{\delta \boldsymbol{\Sigma}}+\mathbf{1}\right)-\widehat{\mathbf{G}}_{\mathbf{U}}[\boldsymbol{\Sigma}]^{-1} \cdot \frac{\delta \widehat{\mathbf{G}}_{\mathbf{U}}[\boldsymbol{\Sigma}]}{\delta \boldsymbol{\Sigma}}
$$

and, using equation (18),

$$
\frac{1}{T} \frac{\delta \widehat{F}_{\mathbf{U}}[\boldsymbol{\Sigma}]}{\delta \boldsymbol{\Sigma}}=-\widehat{\mathbf{G}}_{\mathbf{U}}[\boldsymbol{\Sigma}]
$$

So $\widehat{\mathbf{G}}_{\mathbf{U}}[\boldsymbol{\Sigma}]$ can be considered to be the gradient of the (scalar) self-energy functional $\widehat{F}_{\mathbf{U}}[\boldsymbol{\Sigma}]$. Therewith, the Legendre transform of $\widehat{F}_{\mathbf{U}}[\boldsymbol{\Sigma}]$ can be constructed:

$$
\widehat{\Phi}_{\mathbf{U}}[\mathbf{G}]=\widehat{F}_{\mathbf{U}}\left[\widehat{\boldsymbol{\Sigma}}_{\mathbf{U}}[\mathbf{G}]\right]+\operatorname{Tr}\left(\widehat{\boldsymbol{\Sigma}}_{\mathbf{U}}[\mathbf{G}] \mathbf{G}\right) .
$$

Here $\widehat{\boldsymbol{\Sigma}}_{\mathbf{U}}[\mathbf{G}]$ is the inverse of the functional $\widehat{\mathbf{G}}_{\mathbf{U}}[\boldsymbol{\Sigma}]$. The functional can be assumed to be invertible (locally) provided that the system is not at a critical point for a phase transition (see also [18]). Equation (23) defines the Luttinger-Ward functional. 


\section{Discussion}

\subsection{Properties of the Luttinger-Ward functional}

The properties of the Luttinger-Ward functional, equations (1)-(4), can be easily verified: Equations (10), (14), (19) and (20) imply

$$
\widehat{F}_{\mathbf{U}}\left[\boldsymbol{\Sigma}_{\mathbf{t}, \mathbf{U}}\right]=\Omega_{\mathbf{t}, \mathbf{U}}-\operatorname{Tr} \ln \mathbf{G}_{\mathbf{t}, \mathbf{U}}
$$

and with $\widehat{\mathbf{\Sigma}}_{\mathbf{U}}\left[\mathbf{G}_{\mathbf{t}, \mathbf{U}}\right]=\boldsymbol{\Sigma}_{\mathbf{t}, \mathbf{U}}$ the evaluation of the Luttinger-Ward functional at $\mathbf{G}=\mathbf{G}_{\mathbf{t}, \mathbf{U}}$ yields

$$
\Phi_{\mathbf{t}, \mathbf{U}} \equiv \widehat{\Phi}_{\mathbf{U}}\left[\mathbf{G}_{\mathbf{t}, \mathbf{U}}\right]=\Omega_{\mathbf{t}, \mathbf{U}}-\operatorname{Tr} \ln \mathbf{G}_{\mathbf{t}, \mathbf{U}}+\operatorname{Tr}\left(\boldsymbol{\Sigma}_{\mathbf{t}, \mathbf{U}} \mathbf{G}_{\mathbf{t}, \mathbf{U}}\right),
$$

i. e. equation (1). From equations (22) and (23), one immediately has:

$$
\frac{1}{T} \frac{\delta \widehat{\Phi}_{\mathbf{U}}[\mathbf{G}]}{\delta \mathbf{G}}=\widehat{\mathbf{\Sigma}}_{\mathbf{U}}[\mathbf{G}]
$$

i. e. equation (2). In the limit $\mathbf{U}=0$, the functionals $\widehat{\mathbf{G}}_{\mathbf{U}=0}[\boldsymbol{\Sigma}]$ and $\widehat{F}_{\mathbf{U}=0}[\boldsymbol{\Sigma}]$ are ill-defined (the domain of the functionals shrinks to a single point, $\boldsymbol{\Sigma}=0$, see Appendix A). However, from equation (25), one directly has $\Phi_{\mathbf{U}=0}\left[\mathbf{G}_{\mathbf{t}, 0}\right]=0$ for any $\mathbf{t}$ [see equation (3)] since $\boldsymbol{\Sigma}_{\mathbf{t}, \mathbf{0}}=0$ and $\Omega_{\mathbf{t}, \mathbf{0}}=\operatorname{Tr} \ln \mathbf{G}_{\mathbf{t}, \mathbf{0}}$ (a proof for the latter can be found in [4]). Finally, the universality of $\widehat{\Phi}_{\mathbf{U}}[\mathbf{G}]$, equation (4) is obvious as the definition (23) of the Luttinger-Ward functional involves the universal (t independent) functionals $\widehat{F}_{\mathbf{U}}[\boldsymbol{\Sigma}]$ and $\widehat{\boldsymbol{\Sigma}}_{\mathbf{U}}[\mathbf{G}]$ only.

\subsection{Variational principle}

Using the Legendre transform of the Luttinger-Ward functional $\widehat{F}_{\mathbf{U}}[\boldsymbol{\Sigma}]$, one may define

$$
\widehat{\Omega}_{\mathbf{t}, \mathbf{U}}[\boldsymbol{\Sigma}]=\operatorname{Tr} \ln \frac{1}{\mathbf{G}_{\mathbf{t}, 0}^{-1}-\boldsymbol{\Sigma}}+\widehat{F}_{\mathbf{U}}[\boldsymbol{\Sigma}]
$$

The functional derivative is easily calculated:

$$
\frac{1}{T} \frac{\delta \widehat{\Omega}_{\mathbf{t}, \mathbf{U}}[\boldsymbol{\Sigma}]}{\delta \boldsymbol{\Sigma}}=\frac{1}{\mathbf{G}_{\mathbf{t}, 0}^{-1}-\boldsymbol{\Sigma}}-\widehat{\mathbf{G}}_{\mathbf{U}}[\boldsymbol{\Sigma}]
$$

The equation

$$
\widehat{\mathbf{G}}_{\mathbf{U}}[\boldsymbol{\Sigma}]=\frac{1}{\mathbf{G}_{\mathbf{t}, 0}^{-1}-\boldsymbol{\Sigma}}
$$

is a (highly non-linear) conditional equation for the self-energy of the system $H=H_{0}(\mathbf{t})+H_{1}(\mathbf{U})$ : equations (10) and (19) show that it is satisfied by the exact self-energy $\boldsymbol{\Sigma}=\boldsymbol{\Sigma}_{\mathbf{t}, \mathbf{U}}$. Note that the l.h.s. of (29) is independent of $\mathbf{t}$ but depends on $\mathbf{U}$ (universality of $\widehat{\mathbf{G}}[\boldsymbol{\Sigma}]$ ), while the r.h.s. is independent of $\mathbf{U}$ but depends on $\mathbf{t}$ via $\mathbf{G}_{\mathbf{t}, 0}^{-1}$. The obvious problem of finding a solution of equation (29) is that there is no closed form for the functional $\widehat{\mathbf{G}}_{\mathbf{U}}[\boldsymbol{\Sigma}]$. Solving equation (29) is equivalent to a search for the stationary point of the grand potential as a functional of the self-energy:

$$
\frac{\delta \widehat{\Omega}_{\mathbf{t}, \mathbf{U}}[\boldsymbol{\Sigma}]}{\delta \boldsymbol{\Sigma}}=0 .
$$

Similarly, one can also construct a variational principle using the Green's function as the basic variable, $\delta \widehat{\Omega}_{\mathbf{t}, \mathbf{U}}[\mathbf{G}] / \delta \mathbf{G}=0$. 


\subsection{Dynamical mean-field theory}

The dynamical mean-field theory [14-17] basically applies to lattice models of correlated electrons with on-site interactions such as the Hubbard model [5], for example. The DMFT aims at an approximate solution of equation (29) and is based on two ingredients:

(i) It is important to note that the Luttinger-Ward functional $\widehat{\Phi}_{\mathbf{U}}[\mathbf{G}]$ is the same for the lattice (e. g. Hubbard) model and for an impurity model (single-impurity Anderson model). Actually a (decoupled) set of impurity models has to be considered - one impurity model with the respective local interaction at each site of the original lattice. This ensures that the interaction (U) term is the same as in the lattice model. (In case of translational symmetry the a priori different impurity models can be assumed to be equivalent). As $\mathbf{U}$ is the same in the lattice and in the impurity model, the Luttinger-Ward functional, as well as $\widehat{\mathbf{G}}_{\mathbf{U}}[\boldsymbol{\Sigma}]$, is the same.

(ii) Let the lattice model be characterized by one-particle parameters $\mathbf{t}$ and the impurity model by parameters $\mathbf{t}^{\prime}$. The fundamental equation (29) for the lattice model would then be solved by the exact self-energy $\boldsymbol{\Sigma}_{\mathbf{t}, \mathbf{U}}$. As an ansatz for an approximate solution $\boldsymbol{\Sigma}$ of equation (29), the self-energy is assumed to be local within the DMFT and to be representable as the exact self-energy of the impurity model for some parameters $\mathbf{t}^{\prime}$ :

$$
\boldsymbol{\Sigma}=\boldsymbol{\Sigma}_{\mathbf{t}^{\prime}, \mathbf{U}}
$$

The universality of the Luttinger-Ward functional (i) and the local approximation for the selfenergy (ii) are sufficient to derive the DMFT: The insertion of the ansatz (31) into equation (29) yields a conditional equation for the one-particle parameters of the impurity model $\mathbf{t}^{\prime}$. The l.h.s. becomes $\widehat{\mathbf{G}}_{\mathbf{U}}\left[\boldsymbol{\Sigma}_{\mathbf{t}^{\prime}, \mathbf{U}}\right]=\mathbf{G}_{\mathbf{t}^{\prime}, \mathbf{U}}$, i. e. the exact Green's function of the impurity model, while the r.h.s. reads $\left(\mathbf{G}_{\mathbf{t}, 0}^{-1^{\prime}}-\boldsymbol{\Sigma}_{\mathbf{t}^{\prime}, \mathbf{U}}\right)^{-1}$. The resulting equation for the parameters $\mathbf{t}^{\prime}$ can be fulfilled only locally, i. e. by equating the local elements of the respective Green's functions at the impurity and the original site respectively:

$$
\left(\mathbf{G}_{\mathbf{t}^{\prime}, \mathbf{U}}\right)_{\mathrm{loc}}=\left(\frac{1}{\mathbf{G}_{\mathbf{t}, 0}^{-1}-\boldsymbol{\Sigma}_{\mathbf{t}^{\prime}, \mathbf{U}}}\right)_{\mathrm{loc}} .
$$

This is the so-called self-consistency equation of the DMFT [17].

This consideration can be seen as an independent and, in particular, non-perturbative rederivation of the DMFT which supplements the known approaches such as the cavity method [17].

\subsection{Self-energy-functional approach}

The universality of the Luttinger-Ward functional or of its Legendre transform $\widehat{F}_{\mathbf{U}}[\boldsymbol{\Sigma}]$ is central to the recently developed self-energy-functional approach $[18,19]$. The SFA is a general variational scheme which includes the DMFT as a special limit. The idea is to take as an ansatz for the self-energy of a model $H=H_{0}(\mathbf{t})+H_{1}(\mathbf{U})$ the exact self-energy $\boldsymbol{\Sigma}_{\mathbf{t}^{\prime}, \mathbf{U}}$ of the so-called reference system $H^{\prime}=H_{0}\left(\mathbf{t}^{\prime}\right)+H_{1}(\mathbf{U})$ that shares with the original model the same interaction part. The parameters $\mathbf{t}^{\prime}$ of the one-particle part are considered to be variational parameters to search for the stationary point of the grand potential as a functional of the self-energy. This means to insert the ansatz $\boldsymbol{\Sigma}=\boldsymbol{\Sigma}_{\mathbf{t}^{\prime}, \mathbf{U}}$ into the general expression (27) and to solve the Euler equation $\partial \widehat{\Omega}_{\mathbf{t}, \mathbf{U}}\left[\boldsymbol{\Sigma}_{\mathbf{t}^{\prime}, \mathbf{U}}\right] / \partial \mathbf{t}^{\prime}=0$, i. e.:

$$
\frac{\partial}{\partial \mathbf{t}^{\prime}}\left(\operatorname{Tr} \ln \frac{1}{\mathbf{G}_{\mathbf{t}, 0}^{-1}-\boldsymbol{\Sigma}_{\mathbf{t}^{\prime}, \mathbf{U}}}+\widehat{F}_{\mathbf{U}}\left[\boldsymbol{\Sigma}_{\mathbf{t}^{\prime}, \mathbf{U}}\right]\right)=0
$$

for $\mathbf{t}^{\prime}$. If the search for the optimum set of one-particle parameters $\mathbf{t}^{\prime}$ were unrestricted, the approach would be exact in principle as the Euler equation would then be equivalent to the Euler equation (29) of the general variational principle equation (30). 
A restriction of the space of variational parameters becomes necessary in order to evaluate the quantity $\widehat{\Omega}_{\mathbf{t}, \mathbf{U}}\left[\boldsymbol{\Sigma}_{\mathbf{t}^{\prime}, \mathbf{U}}\right]$ which, in general, is impossible as a closed form for the functional $\widehat{F}_{\mathbf{U}}[\boldsymbol{\Sigma}]$ is not known. With a proper restriction, however, the reference system $H^{\prime}$ can be made accessible to an exact (numerical) solution which makes it possible to derive the exact grand potential and the exact Green's function of the system $H^{\prime}$. Therewith, making use of the universality of $\widehat{F}_{\mathbf{U}}[\boldsymbol{\Sigma}]$ and using equations (23) and (25) for the reference system,

$$
\widehat{F}_{\mathbf{U}}\left[\boldsymbol{\Sigma}_{\mathbf{t}^{\prime}, \mathbf{U}}\right]=\Omega_{\mathbf{t}^{\prime}, \mathbf{U}}-\operatorname{Tr} \ln \mathbf{G}_{\mathbf{t}^{\prime}, \mathbf{U}}
$$

Note that this implies that an exact evaluation of $\widehat{F}_{\mathbf{U}}[\boldsymbol{\Sigma}]$ is possible for self-energies of an exactly solvable reference system with the same interaction part as the original one. Using this result in equation (33), one obtains:

$$
\frac{\partial}{\partial \mathbf{t}^{\prime}}\left(\Omega_{\mathbf{t}^{\prime}, \mathbf{U}}+\operatorname{Tr} \ln \frac{1}{\mathbf{G}_{\mathbf{t}, 0}^{-1}-\boldsymbol{\Sigma}_{\mathbf{t}^{\prime}, \mathbf{U}}}-\operatorname{Tr} \ln \mathbf{G}_{\mathbf{t}^{\prime}, \mathbf{U}}\right)=0
$$

which can be evaluated to fix $\mathbf{t}^{\prime}$ and therewith the optimal self-energy and grand potential (see [18-21] for details and concrete examples).

\subsection{Luttinger's theorem}

Finally, the role of the Luttinger-Ward functional in the derivation of general properties of correlated electron systems will be discussed. As an important example, the Luttinger theorem [4] is considered. For a translationally invariant system, the theorem states that in the limit $T \rightarrow 0$ the average particle number is equal to the volume enclosed by the Fermi surface in $\mathbf{k}$ space:

$$
\langle N\rangle=V_{\mathrm{FS}} .
$$

The Fermi surface is defined by the set of $\mathbf{k}$ points in the first Brillouin zone that satisfies $\mu-\eta_{\mathbf{k}}=0$ where $\eta_{\mathbf{k}}$ are the eigenvalues of the matrix $\mathbf{t}+\boldsymbol{\Sigma}(\omega)$ at the excitation energy $\omega=0$ vanishing. Hence, to formulate the Luttinger theorem, one obviously has to presuppose that there is a Fermi surface at all, i. e. that $\boldsymbol{\Sigma}(\omega=0)$ is Hermitian ${ }^{1}$. The original proof of the theorem [4] is perturbative as it makes use of the skeleton-diagram expansion. A non-perturbative proof, based on topological considerations, was proposed recently [29] and is based on the assumption that the system is a Fermi liquid.

To discuss the Luttinger theorem in the present context, consider the following shift transformation of the Green's function

$$
\mathbf{S}^{(z)} \mathbf{G}\left(\mathrm{i} \omega_{n}\right)=\mathbf{G}^{(z)}\left(\mathrm{i} \omega_{n}\right)=\mathbf{G}\left(\mathrm{i} \omega_{n}+\mathrm{i} z\right)
$$

with $z=2 \pi k T$ and $k$ integer ( $z$ is a bosonic Matsubara frequency). $\mathbf{S}^{(z)}$ is a linear and unitary transformation. The shift transformation leaves the functional integral equation (11) unchanged:

$$
\widehat{\Omega}_{\mathbf{U}}\left[\mathbf{S}^{(z)} \mathbf{G}_{0}^{-1}\right]=\widehat{\Omega}_{\mathbf{U}}\left[\mathbf{G}_{0}^{-1}\right] .
$$

To verify this invariance, one has to note that the shift of the Matsubara frequencies in $\mathbf{G}_{0}^{-1}$ by $z$ can be transformed into a shift $\omega_{n} \rightarrow \omega_{n}-z$ in the Grassmann numbers:

$$
\xi_{\alpha}\left(\mathrm{i} \omega_{n}\right) \rightarrow \xi_{\alpha}\left(\mathrm{i} \omega_{n}-\mathrm{i} z\right)
$$

In imaginary-time representation this shift is equivalent to the multiplication of a phase:

$$
\xi_{\alpha}(\tau) \rightarrow \mathrm{e}^{-\mathrm{i} z \tau} \xi_{\alpha}(\tau), \quad \xi_{\alpha}^{*}(\tau) \rightarrow \mathrm{e}^{\mathrm{i} z \tau} \xi_{\alpha}^{*}(\tau)
$$

\footnotetext{
${ }^{1}$ For systems without Fermi surface, there is no Luttinger theorem. A nice example is given by the Falicov-Kimball model in infinite dimensions, see [28].
} 
This, however, leaves the functional integral unchanged as the transformation (39) or equation (40) is linear and the Jacobian is unity. Note that antiperiodic boundary conditions $\xi_{\alpha}(\tau=1 / T)=$ $-\xi_{\alpha}(\tau=0)$ are respected for a bosonic shift frequency $z$.

Denoting $\Omega_{\mathbf{t}, \mathbf{U}}(z) \equiv \widehat{\Omega}_{\mathbf{U}}\left[\mathbf{S}^{(z)} \mathbf{G}_{\mathbf{t}, 0}^{-1}\right]$, equation (38) states that $\Omega_{\mathbf{t}, \mathbf{U}}(z)=\Omega_{\mathbf{t}, \mathbf{U}}(0)$. Following the steps in the construction of the Luttinger-Ward functional in section 3 , one easily verifies that this implies $\Phi_{\mathbf{t}, \mathbf{U}}(z)=\Phi_{\mathbf{t}, \mathbf{U}}(0)$ where $\Phi_{\mathbf{t}, \mathbf{U}}(z) \equiv \widehat{\Phi}_{\mathbf{U}}\left[\mathbf{S}^{(z)} \mathbf{G}_{\mathbf{t}, \mathbf{U}}\right]$. For the Legendre transform, one has $F_{\mathbf{t}, \mathbf{U}}(z)=F_{\mathbf{t}, \mathbf{U}}(0)$ where $F_{\mathbf{t}, \mathbf{U}}(z) \equiv \widehat{F}_{\mathbf{U}}\left[\mathbf{S}^{(z)} \boldsymbol{\Sigma}_{\mathbf{t}, \mathbf{U}}\right]$. Now, in the limit $T \rightarrow 0, z$ becomes a continuous variable. Hence,

$$
\frac{\mathrm{d}}{\mathrm{d} z} \lim _{T \rightarrow 0} F_{\mathbf{t}, \mathbf{U}}(z)=0 .
$$

If the limit and the derivative can be interchanged,

$$
\frac{\mathrm{d}}{\mathrm{d} z} \lim _{T \rightarrow 0} F_{\mathbf{t}, \mathbf{U}}(z)=\lim _{T \rightarrow 0} \frac{\mathrm{d}}{\mathrm{d} z} F_{\mathbf{t}, \mathbf{U}}(z)
$$

Equations (24) and (41) imply

$$
-\lim _{T \rightarrow 0} \frac{\mathrm{d} \Omega_{\mathbf{t}, \mathbf{U}}(z)}{\mathrm{d} z}=-\lim _{T \rightarrow 0} \frac{\mathrm{d} \operatorname{Tr} \ln \mathbf{S}^{(z)} \mathbf{G}_{\mathbf{t}, \mathbf{U}}}{\mathrm{d} z} .
$$

The $z$ dependence of the grand potential is the same as its $\mu$ dependence, and thus $-(\mathrm{d} / \mathrm{d} z) \Omega_{\mathbf{t}, \mathbf{U}}(z=$ $0)=-\partial \Omega_{\mathbf{t}, \mathbf{U}} / \partial \mu=\langle N\rangle$. The evaluation of the r.h.s. in equation (43) is straightforward and can be found in [4], for example. It turns out that at $z=0$ the r.h.s. is just the Fermi-surface volume $V_{\mathrm{FS}}$.

Consequently, the non-perturbative construction of the Luttinger-Ward functional enables us to reduce the proof of the Luttinger theorem to the proof of equation (42). This, however, requires certain assumptions on the regularity of the $T \rightarrow 0$ limit which are generally non-trivial.

\section{Summary}

To summarize, the present paper has shown that the Luttinger-Ward functional can be constructed within the framework of functional integrals under fairly general assumptions. In particular, there is no need in an adiabatic connection to the non-interacting limit and no expansion is needed in the interaction strength as was required in the original approach by Luttinger and Ward [4]. The construction merely assumes the very existence of the functional integral over Grassmann fields, i. e. the existence of the Trotter limit, for the representation of the partition function.

It is well known that the Luttinger-Ward functional can be employed for different purposes, some of which have been discussed here: The functional is used in order to derive some general properties of correlated electron systems, such as the Luttinger theorem. It enables us to formulate a variational principle involving a thermodynamical potential as a functional of the Green's function or the self-energy and thereby provides a unique and thermodynamically meaningful link between static and dynamic quantities which is helpful for interpretations and for the construction of approximations. An independent derivation of the dynamical mean-field theory is possible using the special properties of the Luttinger-Ward functional and the universality of the functional in particular. The latter is of central importance in the context of the self-energy-functional approach which is a general framework in constructing thermodynamically consistent approximations.

Referring to the standard definition of the Luttinger-Ward functional that is based on the weakcoupling skeleton-diagram expansion, the above-mentioned and any further considerations based on the functional and its unique properties meet with criticism when applied to strongly correlated, non-Fermi liquid or symmetry-broken states. This is exactly the point where the presented nonperturbative construction of the Luttinger-Ward functional is beneficial.

As the proposed functional-integral construction is rather general, one can think of different future extensions: It suggests generalizing the main idea to many-particle Green's functions. For example, the grand potential of an interacting electron system can be expressed as a functional of one- and two-particle Green's functions. The standard construction is diagrammatic again [30] and 
could possibly be replaced by a non-perturbative construction along the lines proposed here. Furthermore, an extension to the non-equilibrium case might be considered. Finally, disordered (and interacting) electron systems deserve further attention. Here an analogous construction is possible which permits to formulate a new class of non-perturbative and thermodynamically consistent approximations. Work along this direction is in progress [31].

\section{Acknowledgements}

The author acknowledges helpful discussions with F.F. Assaad, M. Bechmann, W. Hanke and G. Kotliar. The work is supported by the Deutsche Forschungsgemeinschaft (Forschergruppe 538).

\section{A. Solutions of relation (17)}

As the relation (17) is highly non-linear, the existence and the uniqueness of possible solutions have to be discussed:

Take $\mathbf{U}$ to be fixed and assume that the self-energy given is the exact self-energy of a system $H=H_{0}(\mathbf{t})+H_{1}(\mathbf{U})$ with some hopping parameters $\mathbf{t}$. So the self-energy $\boldsymbol{\Sigma}$ is assumed to be given from the space $\mathcal{S}_{\mathbf{U}}$ of $\mathbf{t}$ representable self-energies $\mathcal{S}_{\mathbf{U}} \equiv\left\{\boldsymbol{\Sigma} \mid \boldsymbol{\Sigma}=\boldsymbol{\Sigma}_{\mathbf{t}, \mathbf{U}}\right.$, t arbitrary $\}$ (U fixed). Using equation (16) it is obvious that the exact Green's function of this system, $\mathbf{G}=\mathbf{G}_{\mathbf{t}, \mathbf{U}}$, solves equation (17) as the Dyson equation (10) shows that $\mathbf{G}_{\mathbf{t}, \mathbf{U}}^{-1}+\boldsymbol{\Sigma}_{\mathbf{t}, \mathbf{U}}$ is the exact free Green's function of this system. Concluding, one has $\widehat{\mathbf{G}}_{\mathbf{U}}\left[\boldsymbol{\Sigma}_{\mathbf{t}, \mathbf{U}}\right]=\mathbf{G}_{\mathbf{t}, \mathbf{U}}$, and thus the existence of a solution is guaranteed on the space $\mathcal{S}_{\mathbf{U}}$. Note that it is very convenient to consider $\mathcal{S}_{\mathbf{U}}$ as the domain of the functional $\widehat{\mathbf{G}}_{\mathbf{U}}[\boldsymbol{\Sigma}]$ since this ensures the correct analytical and causal properties of the variable $\boldsymbol{\Sigma}$.

Under the functional $\widehat{\mathbf{G}}_{\mathbf{U}}[\boldsymbol{\Sigma}]$ the space $\mathcal{S}_{\mathbf{U}}$ is mapped onto the space $\mathcal{G}_{\mathbf{U}}$ of $\mathbf{t}$ representable Green's functions: $\mathcal{G}_{\mathbf{U}} \equiv\left\{\mathbf{G} \mid \mathbf{G}=\mathbf{G}_{\mathbf{t}, \mathbf{U}}, \mathbf{t}\right.$ arbitrary $\}$ (U fixed). In general, the map $\widehat{\mathbf{G}}_{\mathbf{U}}: \mathcal{S}_{\mathbf{U}} \rightarrow$ $\mathcal{G}_{\mathbf{U}}$ is not unique globally. An example is given in [18] (see Appendix A therein). However, the uniqueness of the functional $\widehat{\mathbf{G}}_{\mathbf{U}}[\boldsymbol{\Sigma}]$ can be enforced by a proper restriction of the range $\mathcal{G}_{\mathbf{U}}$, i. e. of the solution set of equation (17). This is explained in detail in [18] (Appendix A). The considerations in sections 3 and 4 are unaffected by such a restriction and hold for any choice of the range. The rest of the discussion in Appendix A of [18] can be applied directly to the present situation. In particular, this shows that there is no general reason to question the local uniqueness of $\widehat{\mathbf{G}}_{\mathbf{U}}[\boldsymbol{\Sigma}]$.

\section{References}

1. Abrikosow A.A., Gorkov L.P., Dzyaloshinski I.E. Methods of Quantum Field Theory in Statistical Physics. Prentice-Hall, New Jersey, 1964.

2. Fetter A.L., Walecka J.D. Quantum Theory of Many-Particle Systems. McGraw-Hill, New York, 1971.

3. Negele J.W., Orland H. Quantum Many-Particle Systems. Addison-Wesley, Redwood City, 1988.

4. Luttinger J.M., Ward J.C., Phys. Rev., 1960, 118, 1417.

5. Hubbard J., Proc. R. Soc. London A, 1963, 276, 238.

6. Baym G., Phys. Rev., 1962, 127, 1391.

7. Luttinger J.M., Phys. Rev., 1960, 119, 1153.

8. Baym G., Kadanoff L.P., Phys. Rev., 1961, 124, 287.

9. Bickers N.E., Scalapino D.J., White S.R., Phys. Rev. Lett., 1989, 62, 961.

10. Bickers N.E., Scalapino D.J., Ann. Phys. (N.Y.), 1989, 193, 206.

11. Keiter H., Kimball J.C., J. Appl. Phys., 1971, 42, 1460.

12. Grewe N., Keiter H., Phys. Rev. B, 1981, 24, 4420.

13. Haule K., Kirchner S., Kroha J., Wölfle P., Phys. Rev. B, 2001, 64, 155111.

14. Metzner W., Vollhardt D., Phys. Rev. Lett., 1989, 62, 324.

15. Georges A., Kotliar G., Phys. Rev. B, 1992, 45, 6479.

16. Jarrell M., Phys. Rev. Lett., 1992, 69, 168.

17. Georges A., Kotliar G., Krauth W., Rozenberg M.J., Rev. Mod. Phys., 1996, 68, 13.

18. Potthoff M., Euro. Phys. J. B, 2003, 32, 429. 
19. Potthoff M., Euro. Phys. J. B, 2003, 36, 335

20. Potthoff M., Aichhorn M., Dahnken C., Phys. Rev. Lett., 2003, 91, 206402.

21. Dahnken C., Aichhorn M., Hanke W., Arrigoni E., Potthoff M., Phys. Rev. B, 2004, 70, 245110.

22. Potthoff M., Adv. Solid State Phys., 2005, 45, 135.

23. Hofstetter W., Kehrein S., Phys. Rev. B, 1999, 59, R12732.

24. Kehrein S., Phys. Rev. Lett., 1998, 81, 3912.

25. Kotliar G., Euro. Phys. J. B, 1999, 11, 27.

26. Savrasov S.Y., Kotliar G., Phys. Rev. B, 2004, 69, 245101.

27. Georges A. Strongly correlated electron materials: Dynamical mean-field theory and electronic structure. Lectures on the Physics of Highly Correlated Electron Systems VIII. American Institute of Physics Conference Proceedings 2004, 715, 3.

28. Freericks J.K., Zlatić V., Rev. Mod. Phys., 2003, 75, 1333.

29. Oshikawa M., Phys. Rev. Lett., 2000, 84, 3370.

30. Almbladh C.O., von Barth U., van Leeuwen R., Int. J. Mod. Phys. B, 1999, 13, 535.

31. Potthoff M., Balzer M. (to be published).

\title{
Непертурбативна побудова функціоналу Люттінгера-Варда
}

\author{
М.Потхоф \\ Інститут теоретичної фізики та астрофізики, Університет Вюрцбург, Хубланд, D-97074 Вюрцбург, \\ Німеччина \\ Отримано 10 лютого 2006 р.
}

\begin{abstract}
Для системи скорельованих електронів функціонал Люттінгера-Варда забезпечує зв'язок між статичними термодинамічними величинами, з одного боку, та одночастинковими збудженнями, з іншого боку. Цей функціонал є придатним для отримання деяких загальних властивостей системи, а також для формулювання термодинамічно узгоджених наближень. Однак його побудова $€$ пертурбативною, оскільки вона базується на діаграмному розкладі при слабкому зв'язку. Показано, що функціонал Люттінгера-Варда можна отримати в рамках загального функціонально-інтегрального підходу. Цей альтернативний непертурбативний підхід підкреслює той факт, що функціонал Лютінгера-Варда $€$ універсальним для широкого класу моделей.
\end{abstract}

Ключові слова: функціонал Люттінгера-Варда, наближення збереження, діаграмна теорія збурень, функціонально-інтегральний підхід, динамічна теорія середнього поля, власноенергетична функціональна теорія

PACS: $71.10 .-w, 71.15 .-m$ 
